\section{Recognition models reevaluated: A response to Smith}

\author{
GIDEON KEREN \\ Institute for Perception TNO \\ Soesterberg, The Netherlands \\ and \\ STAN BAGGEN \\ Institute for Perception Research \\ Eindhoven, The Netherlands
}

The analysis provided by Smith (1982) is an ingenious and illuminating approach to a better understanding of the recognition model that was recently proposed by Keren and Baggen (1981). In that paper, we presented a number of critical comments on the applicability of the Luce $(1959,1963)$ choice model, particularly when applied to recognition of alphanumeric characters. In addition, we developed in that paper another model based on a proposal by Tversky (1977) concerning similarity judgments. The most striking result of Smith's analysis is the formal proof that the feature model proposed by us is mathematically a special case of the general Luce "biased choice" model. This result seems surprising considering the vast difference in points of departure which led to the development of the two models.

Despite the formal equivalence of the two models, we still argue that most of the criticism concerning the applicability of the choice model (presented in our previous article) remains valid. The only critical comment, which we now withdraw, concerns estimation methods. We agree with Smith (1982) that the main problem with zero cells arises from the use of inefficient parameter estimation procedures. The main reason we made that criticism was because previous researchers who have tried to apply the choice model to alphanumeric data have apparently used such inefficient estimation procedures (e.g., Gilmore, Hersh, Caramazza, \& Griffin, 1979; Townsend, 1971). The use of maximum likelihood estimation (Bishop, Fienberg, \& Holland, 1975; Smith, 1980) does, indeed, take care of this problem. In fact, we have also taken Smith's recommendation and have implemented maximum likelihood estimators in our own model.

Our basic disagreement with Smith (1982) concerns the evaluation of models and the comparison

We would like to thank Don Bouwhuis and Charles Lewis for helpful comments on previous drafts of this paper. Requests for reprints should be addressed to Gideon Keren, Institute for Perception TNO, Kampweg 5, Soesterberg, The Netherlands. between competing models. Generally, models can be evaluated on two different dimensions: One is by measures of goodness of fit, that is, the extent to which the model can predict the observed data. A second and separate dimension for evaluating a model concerns its explanatory power, that is, the extent to which we can better understand the phenomenon under investigation by using the proposed model. Both goodness of fit and explanatory power are necessary requirements for the (temporary) acceptance of a model. In other words, in order for a model to be accepted, it has to meet certain criteria on each of the two dimensions. One cannot compensate for inadequacy on one dimension by any level of superiority on the other dimension. In the discussion by Smith (1982), which is entirely focused on one dimension, he argues that the "traditional" " application of the choice model (e.g., as used by Gilmore et al., 1979, and Townsend, 1971) has a better goodness of fit than the KB version, a claim we made in our previous paper; thus, we fully agree with Smith. Our main argument, however, is that the high degree of goodness of fit obtained by the traditional version cannot compensate for its poor explanatory power. Almost any model that utilizes $\mathbf{3 5 0}$ free parameters (the number of free parameters used by the traditional choice model when applied to a confusion matrix with 26 letters) is likely to result in a high degree of goodness of fit, but the question remains as to whether these parameters are interpretable in a meaningful way. As stated in our previous article (Keren \& Baggen, 1981), there are several difficulties in the interpretation of the similarity and response bias matrices that are the outcome of the traditional choice model, and we have serious doubts as to whether such an analysis enhances our understanding of the underlying mechanisms in the recognition process.

Despite the formal identity of the KB model and the choice model, our starting point was completely different. The underlying assumption of our model is that the recognition process (or at least part of it) is composed of feature extraction and feature integration, a topic on which there exists a large body of experimental work. We do not claim that our model is necessarily the "true" model, but that our model is connected to a large body of experimental work and, consequently, can be interpreted in a meaningful way. Obviously, the source for future modifications and elaborations of our model will come from new experimental work on recognition models.

Another advantage of the KB model, when compared with the choice model, is the relatively small number of free parameters, all of which are readily 
interpretable. Thus, although no claim is made that the KB model is the "true" (or "correct") one, we nevertheless believe that it may serve as a promising starting point for future investigation.

In summary, it was asserted that the acceptability of a model should depend on both goodness of fit and explanatory power criteria. Our main argument has been that whereas the KB model meets both types of criteria, the traditional choice model performs very well in terms of the former criteria but fails to meet any reasonable criteria of explanatory power and parsimony.

A large part of Smith's (1982) article is devoted to the comparison of the two models under discussion, and the comparison of the KB model with another special case of the choice model originated by Smith. In the following, we would like to make a few comments on the comparison methods used by Smith (1982).

A generally accepted method for testing whether a set of data is adequately described by a model is the use of a chi-square test. Mostly, the chi-square test is carried out by using the Pearson approximation

$$
\chi^{2} \approx \sum_{\text {all cells }} \frac{(\mathrm{O}-\mathrm{E})^{2}}{\mathrm{E}}
$$

where $O$ and $E$ stand for the observed and expected frequencies, respectively. Another approximation, often used, is the log likelihood ratio defined by

$$
\chi^{2} \approx-2^{\mathrm{e}} \log \left[\frac{1\left(\hat{\theta}_{\mathrm{b}} / \mathrm{D}\right)}{1\left(\hat{\theta}_{\mathrm{a}} / \mathrm{D}\right)}\right],
$$

where $1\left(\hat{\theta}_{\mathrm{a}} / \mathrm{D}\right)$ is the maximum of the likelihood function of model a, given the observed data (D), and $1\left(\hat{\theta}_{\mathrm{b}} / \mathrm{D}\right)$ is the maximum of the likelihood function of model $b$, given the same observed data (D). Note that model $b$ is always a restricted version of model $a$. The $\log$ likelihood ratio is especially suited for the comparison of models.

A major problem of the tests in Equations 1 and 2 arises from the fact that these procedures only approximate the chi-square distribution under the assumption that the models are true. But, obviously, neither the choice model nor the KB model are perfectly true (in fact, they are probably quite far from the truth), and actually any psychological model is likely to be falsified if sufficient data are collected. For instance, if the chi-square is calculated from the choice model on the numeral data (Smith, 1982), this model must be rejected as being not completely true. The comparison of this "nontrue" choice model with a restricted version of it (i.e., the $\mathrm{KB}$ model) on the basis of the log likelihood ratio is therefore meaningless.

Another drawback of comparing models on the basis of the chi-square statistic is its insensitivity to parsimony when the models are fairly simple. For example, assume a confusion matrix of numeral data (Keren \& Baggen, 1981, p. 240 ) and a model that utilizes eight parameters. In such a case, the number of degrees of freedom used for $\chi^{2} / \mathrm{df}$ would be $\mathrm{df}=$ $90-8=82$. Now assume that a modified (restricted) version of this model employs only half the number of parameters (i.e., four) without appreciable degradation in performance. The test for this new version would most likely yield about the same (or worse) chi-square with 86 degrees of freedom, and the improvement would be hardly visible. Parsimony is thus poorly represented in this test, despite its importance as part of the criteria for explanatory power mentioned earlier.

\section{REFERENCES}

Bishop, Y. M. M., Fienberg, S. E., \& Holland, P. W. Discrete multivariate analysis. Cambridge, Mass: M.I.T. Press, 1975.

Gilmore, G. C., Hersh, A., Caramazza, A., \& Griffin, J. Multidimensional letter similarity derived from recognition errors. Perception \& Psychophysics, 1979, 25, 425-431.

Keren, G., \& BAgGen, S. Recognition models of alphanumeric characters. Perception \& Psychophysics, 1981, 29, 234-246.

LUCE, R. D. Individual choice behavior. New York: Wiley, 1959.

LucE, R. D. Detection and recognition. In R. D. Luce, R. R. Bush, \& S. E. Galanter (Eds.), Handbook of mathematical psychology (Vol. 1). New York: Wiley, 1963.

SMITH, J. E. K. Models of identification. In R. Nickerson (Ed.), Attention and performance (Vol. 8). Hillsdale, N.J: Erlbaum, 1980.

Smith, J. E. K. Recognition models evaluated: A commentary on Keren and Baggen. Perception \& Psychophysics, 1982, 31, 183-189.

Townsend, J. T. Theoretical analysis of an alphabetic confusion matrix. Perception \& Psychophysics, 1971, 9, 40-50.

Tversky, A. Features of similarity. Psychological Review, 1977, 84, 327-352.

\section{NOTE}

1. Since Smith has shown that the Keren-Baggen (1981) model is a special case of the choice model, we are now dealing with two versions of the choice model (this does not exclude the existence of other versions): the "traditional" version, in which the probability for confusing any two stimuli is determined by a "similarity" function and a "response bias" function. It has $[n(n+1) / 2]-1$ free parameters ( $n=$ number of stimuli). The second version under discussion is our model which, for brevity, will be referred to as the $\mathrm{KB}$ version.

(Manuscript received January 18, 1982; accepted for publication January 18,1982 .) 